\title{
Origin of strong photoluminescence polarization in GaNP nanowires
}

\author{
Stanislav Filippov, Supanee Sukrittanon, Yanjin Kuang, Charles W. Tu, Per O. Å. Persson, \\ Weimin Chen and Irina Buyanova
}

\section{Linköping University Post Print}

\section{Tweet}

N.B.: When citing this work, cite the original article.

Original Publication:

Stanislav Filippov, Supanee Sukrittanon, Yanjin Kuang, Charles W. Tu, Per O. Å. Persson, Weimin Chen and Irina Buyanova, Origin of strong photoluminescence polarization in GaNP nanowires, 2014, Nano letters (Print), (14), 9, 5264-5269.

http://dx.doi.org/10.1021/n1502281p

Copyright: American Chemical Society http://pubs.acs.org/

Postprint available at: Linköping University Electronic Press http://urn.kb.se/resolve?urn=urn:nbn:se:liu:diva-109932 


\title{
On the origin of strong photoluminescence
}

\section{polarization in GaNP nanowires}

\author{
Stanislav Filippov ${ }^{\dagger}$, Supanee Sukrittanon ${ }^{\S}$, Yanjin Kuang ${ }^{\ddagger}$, Charles Tull, Per O. A. Persson ${ }^{\dagger}$, \\ Weimin M. Chen ${ }^{\dagger}$, and Irina A. Buyanova ${ }^{* \dagger}$ \\ ${ }^{\dagger}$ Department of Physics, Chemistry and Biology, Linköping University, S-581 83 Linköping, \\ Sweden \\ ${ }^{\S}$ Graduate Program of Materials Science and Engineering, La Jolla, California 92093, USA \\ ${ }^{\ddagger}$ of Physics, University of California, La Jolla, California 92093, USA \\ "Department of Electrical and Computer Engineering, University of California, La Jolla, \\ California 92093, USA
}

KEYWORDS: nanowire, photoluminescence, polarization

ABSTRACT. III-V semiconductor nanowires (NWs) have a great potential for applications in a variety of future electronic and photonic devices with enhanced functionality. In this work, we employ polarization resolved micro-photoluminescence ( $\mu$-PL) spectroscopy to study polarization properties of light emissions from individual GaNP and GaP/GaNP core/shell nanowires (NWs) with average diameters ranging between 100 and $350 \mathrm{~nm}$. We show that the near-band-edge emission, which originates from the GaNP regions of the NWs, is strongly 
polarized (up to $60 \%$ at $150 \mathrm{~K}$ ) in the direction perpendicular to the NW axis. The polarization anisotropy can be retained up to room temperature. This polarization behavior, which is unusual for zinc blende NWs, is attributed to local strain in the vicinity of the N-related centers participating in the radiative recombination and to preferential alignment of their principal axis along the growth direction. Our findings therefore show that defect engineering via alloying with nitrogen provides an additional degree of freedom to tailor the polarization anisotropy of III-V nanowires, which is advantageous for their applications as nanoscale emitters of polarized light.

III-V semiconductor nanowires (NWs) are currently attracting increasing research interest owing to their unique electronic and optical properties and a great potential for applications in a variety of future electronic and photonic devices, ranging from optical sensors ${ }^{1}$, photodetectors and solar cells ${ }^{2-4}$, to LEDs, lasers and white lighting ${ }^{5,6}$. Moreover, a reduced constrain in latticematching in the 1D architecture facilitates heterostructure engineering and allows growth of IIIV NWs on foreign (e.g. silicon) substrates, promising for on-chip integration of non-silicon based photonics with silicon nanoelectronics.

Light absorption and emission in thin NWs is expected to be intrinsically anisotropic, i.e. strongly polarized along their axis, due to the so-called antenna effect caused by a large dielectric contrast between a nanowire and its surroundings ${ }^{7,8}$. Consequently, individual III-V NWs can be used as polarization-sensitive photodetectors or nanoscale sources of linearly polarized light ${ }^{2,9}$. This may prove useful in e.g. optically gated switches and highly-dense optical interconnects in photonic-based circuits, where polarization of emission and detection could increase the information bandwidth. For device applications, however, it is desirable to control the polarization direction. This, to a certain extent, can be achieved utilizing selection rules of 
optical transitions. For example, it was recently shown that though polarization of light emission in zinc-blende (ZB) GaAs and InP nanowires is determined by the antenna effect, their wurtzite (WZ) counterparts emit light that is linearly polarized in the direction perpendicular to the NW axis $^{10,11}$. The WZ phase formation, however, is energetically favorable only in thin $\mathrm{NWs}^{12}$ with a high surface-to-volume ratio, which imposes size restrictions and also limits maximum achievable polarization in $\mathrm{WZ}$ wires due to the counteracting optical antenna effect ${ }^{13}$.

Previous polarization studies of III-V NWs were mainly focused on InP- and GaAs- based compounds used in today's optoelectronics and photonics. A novel material system with a great potential for these applications is GaNP alloys. Isoelectronic replacement of phosphorous by several percentages of nitrogen leads to a giant reduction in the bandgap energy of nitrogen alloyed gallium phosphide due to the bowing effect and also causes a change in the bandgap character from an indirect one in GaP to a quasi-direct bandgap in GaNP alloys with [N] $>0.4 \%$ 14-16. This enhances the oscillator strength of optical transitions, making this material suitable for highly-efficient yellow-amber LEDs and white lighting ${ }^{17}$. Furthermore, since the lattice constant of GaP decreases upon N incorporation, GaNP can be lattice-matched to Si. This is advantageous for integration of the main-stream Si-based electronics with optoelectronics dominated by III-V compounds. Feasibility of the growth of co-axial GaP/GaNP NWs by molecular beam epitaxy (MBE) on Si substrates was demonstrated most recently ${ }^{18}$. The wires were concluded to have an excellent optical quality, evident from the observation of bright room-temperature emission from a single $\mathrm{NW}^{19}$.

In this work we investigate polarization properties of such GaNP NWs and show that alloying with nitrogen allows one to achieve strong polarization of light emission perpendicular to the 
wire axis even in ZB nanowires of various diameters. The observed polarization property is attributed to strong anisotropy of N-related centers responsible for the emission.

The investigated GaNP NWs were grown by MBE on (111) Si substrates as described in detail elsewhere $^{18}$. In order to analyze effects of growth conditions and nanowire geometry on polarization of the emitted light, three sets of structures were chosen. These include: (i) GaNP NWs with a typical diameter of around $100 \mathrm{~nm}$; (ii) GaP/GaNP core/shell NWs with a GaP core diameter of $110 \mathrm{~nm}$ and a total diameter (d) of $220 \mathrm{~nm}$; and (iii) $\mathrm{GaP} / \mathrm{Ga}_{1-\mathrm{x}} \mathrm{N}_{\mathrm{x}} \mathrm{P} / \mathrm{Ga}_{1-\mathrm{y}} \mathrm{N}_{\mathrm{y}} \mathrm{P}$ core/shell/shell NWs with $\mathrm{x}>\mathrm{y}$, with the core diameter of $110 \mathrm{~nm}$ and the shell thicknesses of 50 $\mathrm{nm}$ and $80 \mathrm{~nm}$. The GaNP NWs and the GaP core in the core/shell structures were grown via the vapor-liquid-solid (VLS) mechanism whereas the growth of the GaNP shell layers was stepmediated $^{18}$.

According to the previous scanning electron microscopy (SEM) measurements performed on the same structures ${ }^{18}$, the wires exhibit a hexagonal cross-section, indicating that they were epitaxially grown along the [111] direction. The crystal structure of the nanowires with different diameters was further analyzed using transmission electron microscopy (TEM). All wires are found to be single crystalline predominantly with ZB lattice structure, but have a rather high density of mirror planes oriented perpendicularly to the growth direction. As an example, figure 1a shows an overview TEM image of a GaP/GaNP NW obtained using a double corrected $\operatorname{Titan}^{3}$ 60-300 microscope. The corresponding high-resolution TEM image is presented in figure 1b and reveals the ZB structure of the NW, as evident from the fast Fourier transform (inset), and twin formation. 

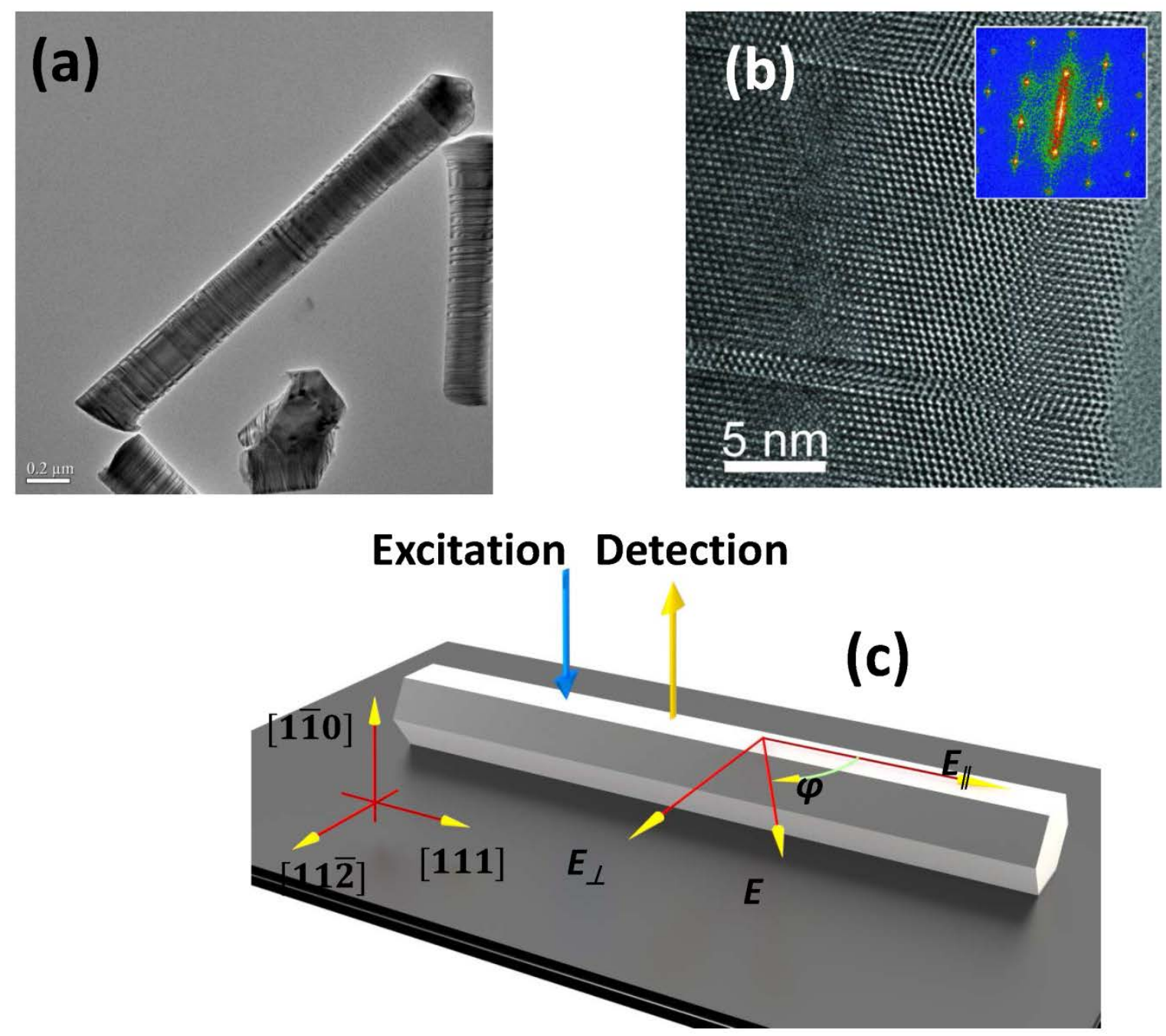

Figure 1. (a) Representative overview TEM image of a GaNP nanowire. (b) Atomically resolved image of a NW edge, exhibiting the ZB structure as evident from the fast Fourier transform (inset) and revealing the randomly occurring twins. (c) Schematic diagram of the measurement geometry utilized for polarization resolved $\mu$-PL measurements. The Cartesian coordinate system is chosen according to the nanowire crystal directions and geometry. $\boldsymbol{E}$ denotes the polarization direction of the analyzer rotated by the angle $\varphi$ relative to the nanowire axis, with $\boldsymbol{E}_{\|}$ and $\boldsymbol{E}_{\perp}$ being parallel and perpendicular to the NW axis, respectively. 
Polarization properties of the light emission were analyzed by employing microphotoluminescence ( $\mu-\mathrm{PL})$ spectroscopy performed on a single NW. For these purposes, the wires were mechanically transferred onto another Si substrate and mounted inside a cold finger cryostat fixed to an $\mathrm{X}-\mathrm{Y}$ translational stage. The PL spectra were measured using a confocal Horiba Jobin-Yvon HR800 system in the backscattering configuration. A 50x objective lens with $\mathrm{NA}=0.5$ was used for both focusing the excitation light from a $445 \mathrm{~nm}$ diode laser and collecting the emitted PL. Typical excitation power density was around $30 \mu \mathrm{W} / \mu \mathrm{m}^{2}$. The PL signal was dispersed by an 800 mm grating monochromator and detected by a charge-coupleddevice (CCD) detector. For polarization-resolved PL measurements, a polarization analyzer that consisted of a rotatable $\lambda / 2$-retardation plate and a fixed linear polarizer (Glan-Thompson prism) was placed in the detection path before the entrance slit of the monochromator. The polarization optics was achromatic within the spectral range of interest. In addition, a depolarizer was inserted in the excitation path to eliminate any effects of optical orientation. The PL intensity (I) was measured as a function of the angle $(\varphi)$ between the polarization axis of the analyzer (E) and the nanowire axis as shown schematically in figure 1c. The degree of linear polarization of PL was calculated by $P=100\left(I_{\|}-I_{\perp}\right) /\left(I_{\|}+I_{\perp}\right)$, where $\mathrm{I}_{\|}\left(\mathrm{I}_{\perp}\right)$ denotes the PL intensity detected in linear polarization parallel (perpendicular) to the direction of the NW axis.

Typical near-bandedge PL spectra recorded at $150 \mathrm{~K}$ from individual GaP, GaP/GaNP and $\mathrm{GaP} / \mathrm{Ga}_{1-\mathrm{x}} \mathrm{N}_{\mathrm{x}} \mathrm{P} / \mathrm{Ga}_{1-\mathrm{y}} \mathrm{N}_{\mathrm{y}} \mathrm{P}$ NWs with total diameters of 100,220 and $350 \mathrm{~nm}$, respectively, are shown in the upper part of figures 2a - 2c. The spectra are dominated by a rather broad PL band due to recombination of excitons trapped at various N-related localized states within the GaNP regions $^{19}$. In the case of $\mathrm{GaP} / \mathrm{Ga}_{1-\mathrm{x}} \mathrm{N}_{\mathrm{x}} \mathrm{P} / \mathrm{Ga}_{1-\mathrm{y}} \mathrm{N}_{\mathrm{y}} \mathrm{P}$ structures, the $\mathrm{PL}$ transitions occur in the inner $\mathrm{Ga}_{1-\mathrm{x}} \mathrm{N}_{\mathrm{x}} \mathrm{P}$ shell, as a result of fast trapping of photoexcited carriers from the adjacent GaP core 
and the outer $\mathrm{Ga}_{1-\mathrm{y}} \mathrm{N}_{\mathrm{y}} \mathrm{P}$ shell with wider bandgaps. The PL peak position varies only slightly between these structures, indicating similar nitrogen compositions in the emitting GaNP shells.
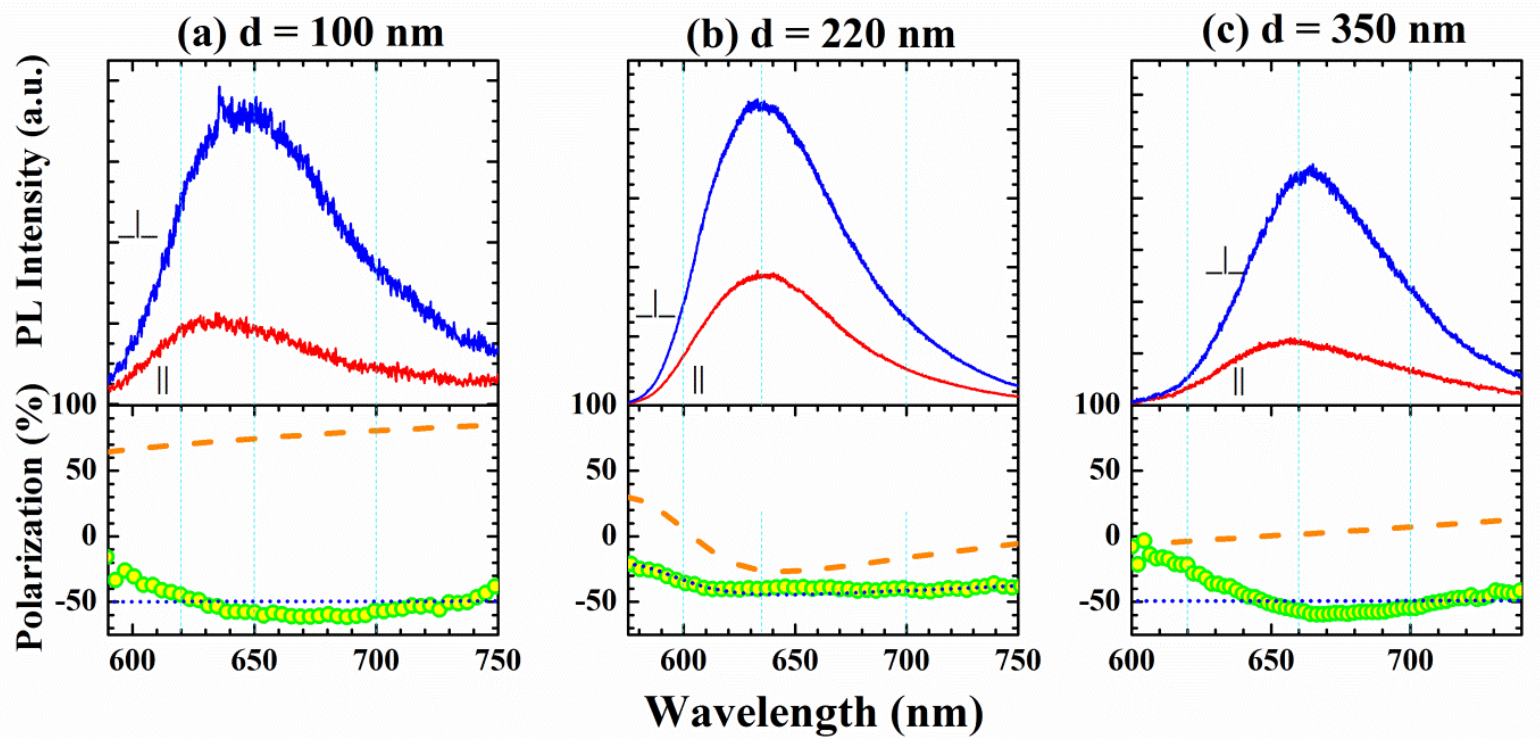

Figure 2. Typical PL spectra (solid lines) and linear polarization degree (open circles, green online) measured at $150 \mathrm{~K}$ from a GaNP NW (a), GaP/GaNP core/shell NW (b) and GaP/GaNP/GaNP core/shell/shell NW (c) with an average diameter of $100 \mathrm{~nm}, 220 \mathrm{~nm}$, and 350 $\mathrm{nm}$, respectively. Labels $\|$ and $\perp$ denote the PL spectra obtained by detecting linear polarizations parallel and perpendicular to the NW axis, respectively. The dashed and dotted lines are simulated spectral dependences of $\mathrm{P}$ expected from the antenna effect for an isotropic and anisotropic emitter, respectively. In the latter case, the degree of linear polarization inside the NW was assumed to be equal to $-96 \%$ (a), $-43 \%$ (b) and $-82 \%$ (c), respectively. The vertical dashed lines denote the PL wavelengths used for further analysis shown in figures 3 and 4.

The PL signal in all structures was found to be substantially stronger when it was detected in the linear polarization perpendicular to the direction of the NW axis as compared with that 
parallel to the NW axis, which is rather surprising taking into account their ZB structure. This unusual perpendicular polarization of the emitted light is further confirmed from polar plots of the PL intensity measured as a function of an analyzer angle with respect to the NW axis. The corresponding data are shown by symbols in figure 3 for several emission wavelengths. They can be fitted (the solid lines) by $I=I_{\min } \sin ^{2}(\varphi-\theta)+I_{\max } \cos ^{2}(\varphi-\theta)$, where $I_{\max }\left(I_{\min }\right)$ defines the maximum (minimum) PL intensity and $\theta$ corresponds to the angle with respect to the NW axis at which the PL intensity reaches $\mathrm{I}_{\max }$. The best fit to the data yields $\theta=90 \pm 10^{\circ}$, which means that $\mathrm{I}_{\perp}\left(\mathrm{I}_{\mathrm{I}}\right) \approx \mathrm{I}_{\max }\left(\mathrm{I}_{\min }\right)$. Spectral dependencies of the linear polarization $\mathrm{P}$ can then be calculated from the PL spectra presented in the upper part of figure 2 and are shown by the open circles in the lower part of the figure 2. The polarization degree is found to be rather high and falls into the range between -40 and $-60 \%$. We note that these values are similar to those reported for $\mathrm{WZ}$ GaAs and InP that were obtained at noticeably lower temperatures ${ }^{10,11}$. P remains negative for all emission wavelengths though its value decreases at the high energy side of the PL spectra. This leads to a spectral shift of the PL maximum positions between the two orthogonal polarizations as seen from figure 2, as the longer-wavelength PL components are more polarized and, therefore, lead to a stronger contribution to the PL spectra measured in the linear polarization perpendicular to the NW axis. 


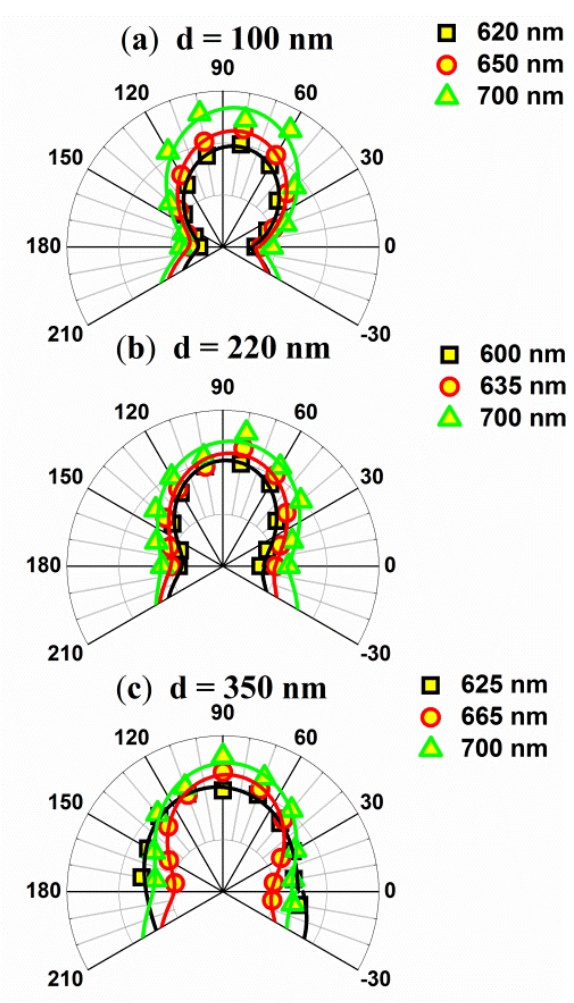

Figure 3. Polar plots of the PL intensity measured at $150 \mathrm{~K}$ for the specified detection wavelengths from an individual GaNP NW (a), GaP/GaNP core/shell NW (b), and GaP/GaNP/GaNP core/shell/shell NW (c).

It is known that polarization properties of NWs with diameters smaller than the PL wavelength could be modified by the antenna effect to an extent determined by the NW diameter and emission wavelength ${ }^{7,8}$. In order to evaluate implications of this effect in the studied NWs, we performed simulations of the expected PL polarization caused by the dielectric mismatch between the GaNP and the ambient air. The simulations were done following the formalism developed in Refs. 5 and 6, assuming that the incorporation of several percent of nitrogen (i.e. below $3 \%$ ) in GaP does not substantially affect its refractive index. Spectral dependence of the refractive index of GaP was taken from Ref. 20. The results of these calculations for isotropic 
internal emission are shown by the dashed lines in the low part of figure 2 and are in obvious disagreement with the experimental data. To improve agreement with the experiment we need to assume that the PL light emitted inside the nanowire is strongly polarized perpendicularly to the NW axis. From the best fit to the experimental data, the linear polarization degree of the PL inside the NWs can then be estimated as being about $-96 \%,-43 \%$ and $-82 \%$ for the NWs with $d$ = 100, 220 and $350 \mathrm{~nm}$, respectively. The internal PL polarization seems to be higher for the thinner NWs grown via the VLS mechanism than for the core/shell structures, where the active GaNP shell was fabricated via the step-mediated growth. One also notices that the antenna effect could not fully account for the observed spectral dependence of the PL polarization with a decreased polarization anisotropy at the high energy side of the PL spectra.

The described spectral behavior of the PL polarization is observed at all measurement temperatures over the range of $4-300 \mathrm{~K}$. This is demonstrated in figure 4 which shows temperature variation of the angle $\theta$ (figures $4 \mathrm{a}-4 \mathrm{c}$ ) and the PL polarization degree (figures $4 \mathrm{~d}-$ 4f) determined at several emission wavelengths. One notices that though the polarization direction of the emitted light stays perpendicular to the axes of the NWs, the polarization degree somewhat increase at low $\mathrm{T}$. This effect is more pronounced for the higher energy regions of the PL spectra - see e.g. figures $4 e$ and $4 f$. 

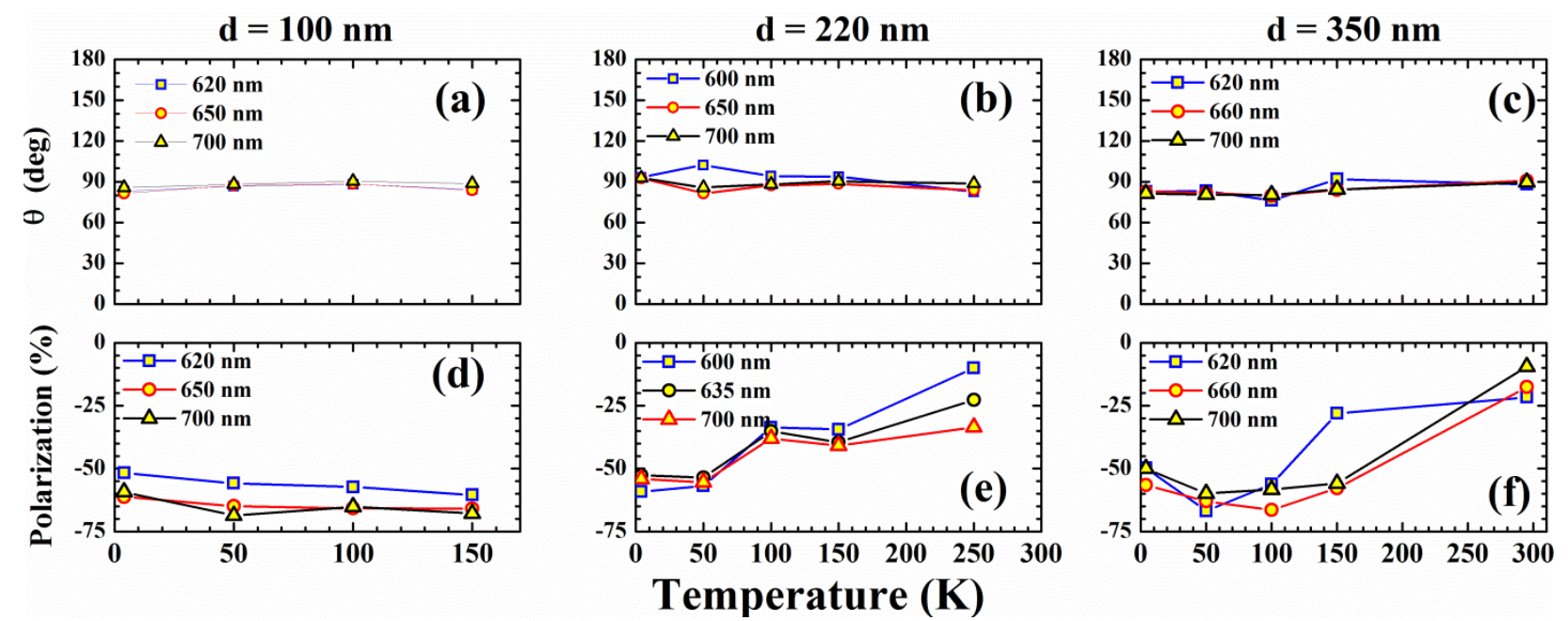

Figure 4. Temperature dependences of the angle $\theta$ at which the maximum PL intensity was observed (a -c) and the linear polarization degree $P(\mathrm{~d}-\mathrm{f})$, measured at the specified detection wavelengths. (For the NWs with $\mathrm{d}=100$ (220) nm the PL polarization was measured at $\mathrm{T} \leq 150$ (250K) K, due to quenching of the PL intensity at higher temperatures).

Let us now discuss the origin of the observed PL polarization that is rather unusual for thin $\mathrm{ZB}$ nanowires. We first recall that the PL emission from GaNP NWs is governed by recombination of excitons trapped at various N-related centers ${ }^{19}$. This mechanism is not unique to the NW geometry but is common in GaNP alloys where increasing $\mathrm{N}$ content is known to cause transformation of the PL spectra from a series of sharp lines emitting very close to the bandgap energy of GaP to a deeper broad PL band ${ }^{21-23}$. The former are related to excitons bound to pairs of $\mathrm{N}$ atoms (NN pairs) with different spatial separation, of which the binding energy decreases with increasing pair separation ${ }^{17}$. The latter is formed by overlapping transitions from deeper $\mathrm{N}$ related cluster states.

Electronic structure of isolectronic excitons bound to such complex centers is determined by combined effects of local field and exchange interaction (or J-J coupling) between a $\mathrm{S}_{1 / 2}$-like 
electron and a $\mathrm{P}_{3 / 2}$-like hole forming the exciton ${ }^{21,24,25}$. At sufficiently low temperatures an optical transition from each individual center can be polarized as determined by its local symmetry. As an example, we can consider a polarization response of an axial center with the symmetry of $\mathrm{C}_{3 \mathrm{v}}$ or higher. The axial crystal field, which resembles uniaxial strain, will lift the degeneracy of the $\mathrm{P}_{3 / 2}$-like hole states but would not affect the electron state. The J-J coupling will further split the excitonic states according to the projection $\mathrm{m}_{\mathrm{J}}$ of the total angular momentum $\mathrm{J}$ along the local symmetry axis ${ }^{24,25}$. The energy states of bound excitons (BE) for compressive and tensile local strain are shown schematically in figures 5a and 5b, respectively. In the case when the strain splitting $(2 \varepsilon)$ of the hole substates exceeds the electron-hole exchange splitting $\Delta$, the exciton energy levels are composed of two groups of states which are separated in energy comparable with $2 \varepsilon$. From figure 5 it is obvious that independent of the sign of the local strain, the PL light emitted at low temperatures from a single center should be completely polarized in the plane perpendicular to the center's principle axis. (Lowering down the symmetry will lift the remaining degeneracy and could change the ordering of the exciton states, but may retain polarization of optical transitions from individual excitonic sublevels.) 
(a) Compression

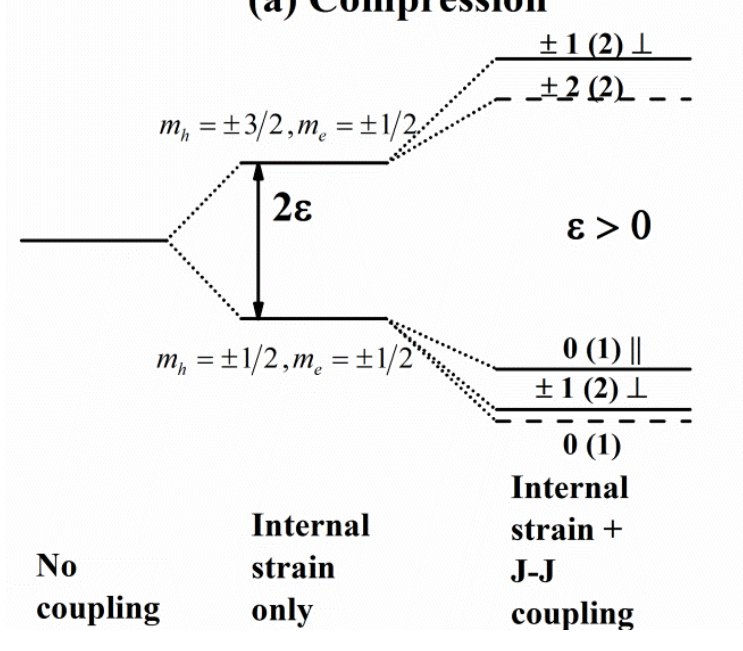

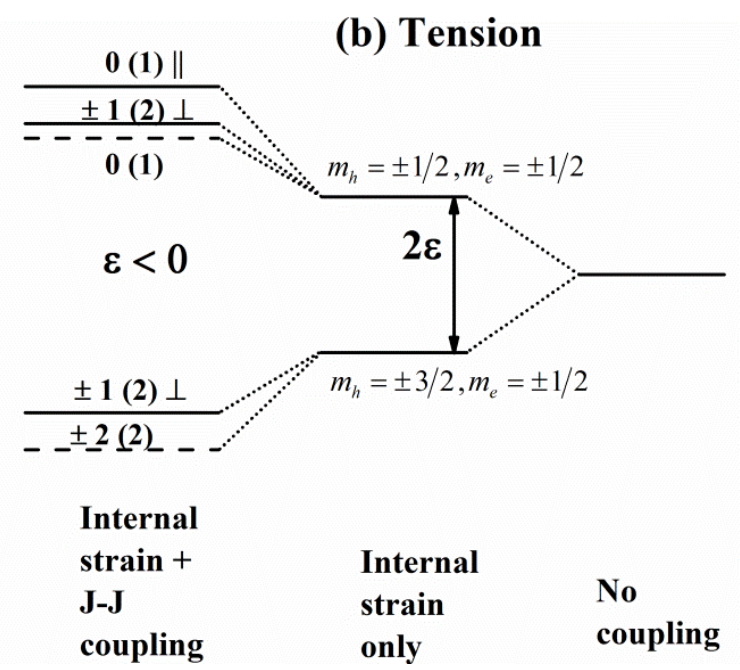

Figure 5. Electronic states of an exciton bound to an isoelectronic center with symmetry of $\mathrm{C}_{3 \mathrm{v}}$ or higher in the presence of (a) compressive and (b) tensile local strain. The local strain is assumed to dominate over exchange interaction. The solid (dashed) lines represent the states corresponding to dipole allowed (forbidden) optical transitions. The unbracketed numbers are the $m_{J}$ values of the exciton sublevels whereas the bracketed numbers indicate their degeneracy. The polarization of the allowed transitions is indicated relative to the principle axis of the center.

In principle, the polarization anisotropy should be reduced if one takes into account orientational degeneracy of the centers. To evaluate the extent of this effect, we have calculated a polarization response of the PL light emitted from anisotropic centers with their principle axes orientated along several crystallographic directions with the lowest number of equivalent orientations. The corresponding angular dependencies of the PL intensity are shown in figures 6a, 6b, 6c and 6d, taking as an example <110>, <001>, <211> and <111> crystallographic directions, respectively. The solid (dotted) lines represent simulation results obtained assuming that the light emitted from an individual center is polarized perpendicular (parallel) to its principle axis. From figure 6 it is apparent that though some polarization anisotropy could be 
retained, it is by far too weak to account for the experimental findings shown in figure 3 . For the centers with lower symmetry, i.e. oriented along the high-index crystallographic directions, an increasing number of equivalent orientations leads to further depolarization. The high degree of the PL polarization in all studied wires, therefore, suggests that the N-related centers responsible for the NW emission are not orientation degenerate but possess a particular symmetry axis determined by the growth process. This axis should be oriented so that the emitted light is polarized in the plane perpendicular to the NW axis.

The experimental results can be consistently explained if we assume that the principle axis of the N-related centers coincides with the [111] growth direction of the NWs. The electronic structure of the related BE and its polarization then follow the scheme shown in figure 5 . At cryogenic temperatures, preferential population of the $\mathrm{m}_{\mathrm{j}}= \pm 1$ exciton state will lead to complete PL polarization in the plane orthogonal to the NW direction. This strong PL polarization should be observed independent of the sign of the local strain field. Expected temperature behavior of the polarization degree, however, differs significantly depending on the sign of the axial local crystal field at the $\mathrm{N}$-related centers. In the case of compressive strain (see figure $5 \mathrm{a}$ ), a temperature increase should first lead to population of the $m_{j}=0$ exciton state accompanied by a decrease of polarization degree from $-100 \%$ down to $-33 \%$, when both $m_{j}= \pm 1$ and $m_{j}=0$ states become equally populated. Since the J-J coupling energy $\Delta$ for N-related BE in GaP is of the order of $1 \mathrm{meV}^{20,23,24}$, this decrease in the PL polarization should be observed at rather low measurement temperatures. Polarization anisotropy, however, is expected to be partly restored at higher temperatures due to thermal population of the topmost $\mathrm{m}_{\mathrm{j}}= \pm 1$ state. Such nonmonotonous temperature dependence of $\mathrm{P}$ will not be observed if the local distortion around the $\mathrm{N}$-center results in tensile local strain (see figure 6b). Here, the depolarization of PL will appear 
only when the topmost $m_{j}=0$ state becomes populated. Therefore, depending on the strength of the tensile strain and, consequently, the $\varepsilon / \Delta$ ratio, one will observe that the degree of polarization either remains constant or gradually decreases with increasing $\mathrm{T}$.
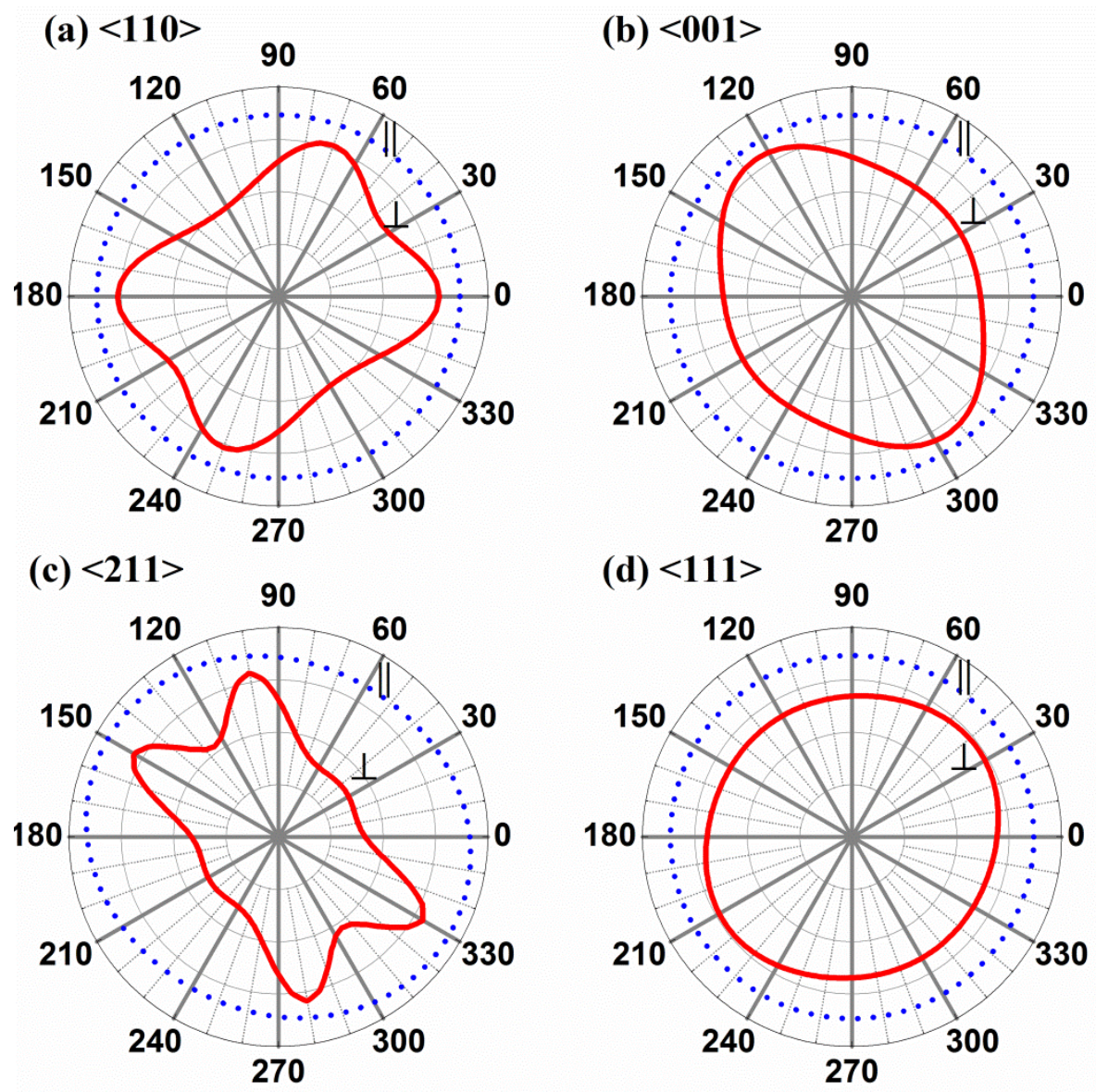

Figure 6. Calculated polar patterns of the PL intensity from orientation degenerate anisotropic emitters oriented along <110> (a), <001> (b), <211> (c), and <111> (d) crystallographic directions. The solid red (dashed blue) curves correspond to the case when the light emitted from an individual anisotropic center is polarized in the plane perpendicular to its principal axis (along the principal axis). 
From figure 4 it is apparent that the experimental data follows the second scenario, i.e. that the $\mathrm{N}$-related centers experience tensile local strain. This is probably not surprising, considering a considerably smaller covalent radius of an $\mathrm{N}$ atom as compared with the replaced $\mathrm{P}$ atom. The measured temperature dependences of the PL polarization degree also suggest that the local strain field can be somewhat affected by the growth conditions and is lower for the shallower Nrelated centers that are responsible for light emission at the high energy side of the PL spectra. This leads to a more pronounced depolarization of the related PL components at elevated temperatures. We therefore suggest that the strong orthogonal PL polarization in the studied NWs reflects the local structure of the N-related cluster states responsible for the BE emission. Specifically, a majority of these centers experience local tensile strain so that their principle axis is orientated along the NW axis, i.e. the clusters are preferentially aligned in one direction. This effect is common for all NWs irrespective of the growth mechanism responsible for the formation of the GaNP layer. On average, however, the degree of this alignment seems to be higher when the GaNP regions are grown via the VSL mechanism as compared to that when the growth is step mediated. This is based on a higher degree of linear PL polarization inside the GaNP NWs as compared with that in the GaP/GaNP core/shell or GaP/GaNP/GaNP core/shell/shell structures. Though the origin of this alignment can not be unambiguously established from the present study, a possible reason could be a high density of twins orthogonal to the growth direction that affect the local structure of the N-related centers.

In summary, we have employed polarization resolved $\mu$-PL spectroscopy to study polarization properties of light emission from GaNP NWs and related axial and co-axial structures. We showed that in spite of the ZB lattice structure, the near-bandedge PL emission in these NWs is 
strongly polarized (up to $60 \%$ at $150 \mathrm{~K}$ ) in the direction perpendicular to the NW axis. The polarization anisotropy can be achieved in the wires of various diameters (i.e. 100 - $350 \mathrm{~nm}$ ) and is retained up to room temperature. This polarization response, which is unusual for ZB NWs, is attributed to the local strain in the vicinity of the N-related centers participating in the radiative recombination and to the preferential alignment of their principal axis along the growth direction, presumably caused by the presence of planar defects. Our findings therefore show that defect engineering via alloying with nitrogen provides an additional degree of freedom to control the polarization anisotropy of III-V nanowires, advantageous for their applications as a nanoscale source of polarized light.

\section{AUTHOR INFORMATION}

\section{Corresponding Author}

*E-mail: iribu@ifm.liu.se

\section{Author Contributions}

The samples were grown by S.S., Y.K. under supervision of C. W. Tu. TEM images were obtained by P.O.Å.P. S.F. carried out the $\mu$-PL measurements and analyzed the data with guidance from W.M.C. and I.A.B. I.A.B. wrote the final version of the manuscript, with contributions from the co-authors.

\section{Acknowledgement}

Financial support by the Swedish Research Council (grant \# 621-2010-3815) is greatly appreciated. The nanowire growth is supported by the U.S. National Science Foundation under Grant \# DMR-0907652 and DMR-1106369. S.S. is partially funded by a Royal Government of Thailand Scholarship. The Linköping double corrected TEM microscope was funded by the Knut 
and Alice Wallenberg Foundation. The authors are grateful to A. Dobrovolsky for his help at early stages of this work.

\section{REFERENCES}

(1) Sirbuly, D. J.; Tao, A.; Law, M.; Fan, R.; Yang, P. Adv. Mater. 2007, 19, 61-66.

(2) Wang, J.; Gudiksen, M.S.; Duan, X.; Cui, Y.; Lieber, C.M. Science 2001, 293, 14551457.

(3) Tian, B.; Zheng, X.; Kempa, T. J.; Fang, Y.; Yu, N.; Yu, G.; Huang, J.; Lieber, C. M. Nature 2007, 449, 885-889.

(4) Wallentin, J.; Anttu, N.; Asoli, D.; Huffman, M.; Åberg, I.; Magnusson, M. H.; Siefer, G.; Fuss-Kailuweit, P.; Dimroth, F.; Witzigmann, B. et al. Science 2013, 339, 1057-1060.

(5) Minot, E. D.; Kelkensberg, F.; van Kouwen, M.; van Dam, J. A.; Kouwenhoven, L. P.; Zwiller, V.; Borgström, M. T.; Wunnicke, O.; Verheijen, M. A.; Bakkers, E. P. A. M. Nano Lett. 2007, 7, 367-371.

(6) Duan, X.; Huang, Y.; Agarwal, R.; Lieber, C. M. Nature 2003, 421, 241-245.

(7) Ruda, H. E.; Shik A. J. Appl. Phys. 2006, 100, 024314.

(8) Ruda, H.; Shik, A. Phys. Rev. B. 2005, 72, 115308.

(9) Muskens, O. L.; Diedenhofen, S. L.; van Weert, M. H. M.; Borgström, M. T.; Bakkers, E. P. A. M.; Gomez, J.R. Adv. Funct. Mater. 2008, 18, 1039-1046. 
(10) Mishra, A.; Titova, L. V.; Hoang, T. B.; Jackson, H. E.; Smith, L. M.; Yarrison-Rice, J. M.; Kim, Y.; Joyce, H. J.; Gao, Q.; Tan, H. H.; Jagadish, C. Appl. Phys. Lett. 2007, 91, 263104.

(11) Hoang, B. T.; Moses, A. F.; Ahtapodov, L.; Zhou, H.; Dheeraj, D. L.; van Helvoort, A. T. J.; Fimland, B-O.; Weman, H. Nano Lett. 2010, 10, 2927-2933.

(12) Caroff, P.; Dick, K. A.; Johansson, J.; Messing, M. E.; Deppert, K.; Samuelson, L. Nature Nanotech. 2008, 4, 50-55.

(13) Joyce, H. J.; Gao, Q.; Hoe Tan, H.; Jagadish, C.; Kim, Y.; Zou, J.; Smith, L. M.; Jackson, H. E.; Yarrison-Rice, J. M.; Parkinson, P. et al. Prog. Qaunt. Eelectron. 2011, 35, 23-75.

(14) Shan, W.; Walukiewicz, W.; Yu, K. M.; Wu, J.; Ager, J. W.; Haller, E. E.; Xin, H. P.; Tu, C. W. Appl. Phys. Lett. 2000, 76, 3251.

(15) Xin, H. P.; Tu, C. W.; Zhang, Y.; Mascarenhas, A. Appl. Phys. Lett. 2000, 76, 1267.

(16) Buyanova, I. A.; Pozina, G.; Bergman, J. P.; Chen, W. M.; Xin, H. P.; Tu, C. W. Appl. Phys. Lett. 2002, 81, 52.

(17) Odnoblyudov, V. A.; Tu, C. W. J. Vac. Sci. Technol. B. 2006, 24, 2202.

(18) Kuang, Y. J.; Sukrittanon, S.; Li, H.; Tu, C. W. Appl. Phys. Lett. 2012, 100, 053108.

(19) Dobrovolsky, A.; Stehr, J. E.; Chen, S. L.; Kuang, Y. J.; Sukrittanon, S.; Tu, C. W.; Chen, W. M.; Buyanova, I. A. Appl. Phys. Lett. 2012, 101, 163106.

(20) Aspens, D.E.; Studna, A. A. Phys. Rev. B. 1983, 27, 985.

(21) Thomas, D. G.; Hopfield, J. J. Phys. Rev. Lett. 1966, 313, 680. 
(22) Liu, X.; Bishop, S. G.; Baillargeon, J. N.; Cheng, K. Y. Appl. Phys. Lett. 1993, 63, 208.

(23) Buyanova, I. A.; Rudko, G. Yu.; Chen, W. M.; Xin, H. P.; Tu, C. W. Appl. Phys. Lett. 2002, 80, 1740 .

(24) Morgan, J. W.; Morgan, T. N. Phys. Rev. B. 1970, 1, 739.

(25) Gil, B.; Camassel, J.; Merle, P.; Mathieu, H. Phys. Rev. B. 1982, 25, 3987.

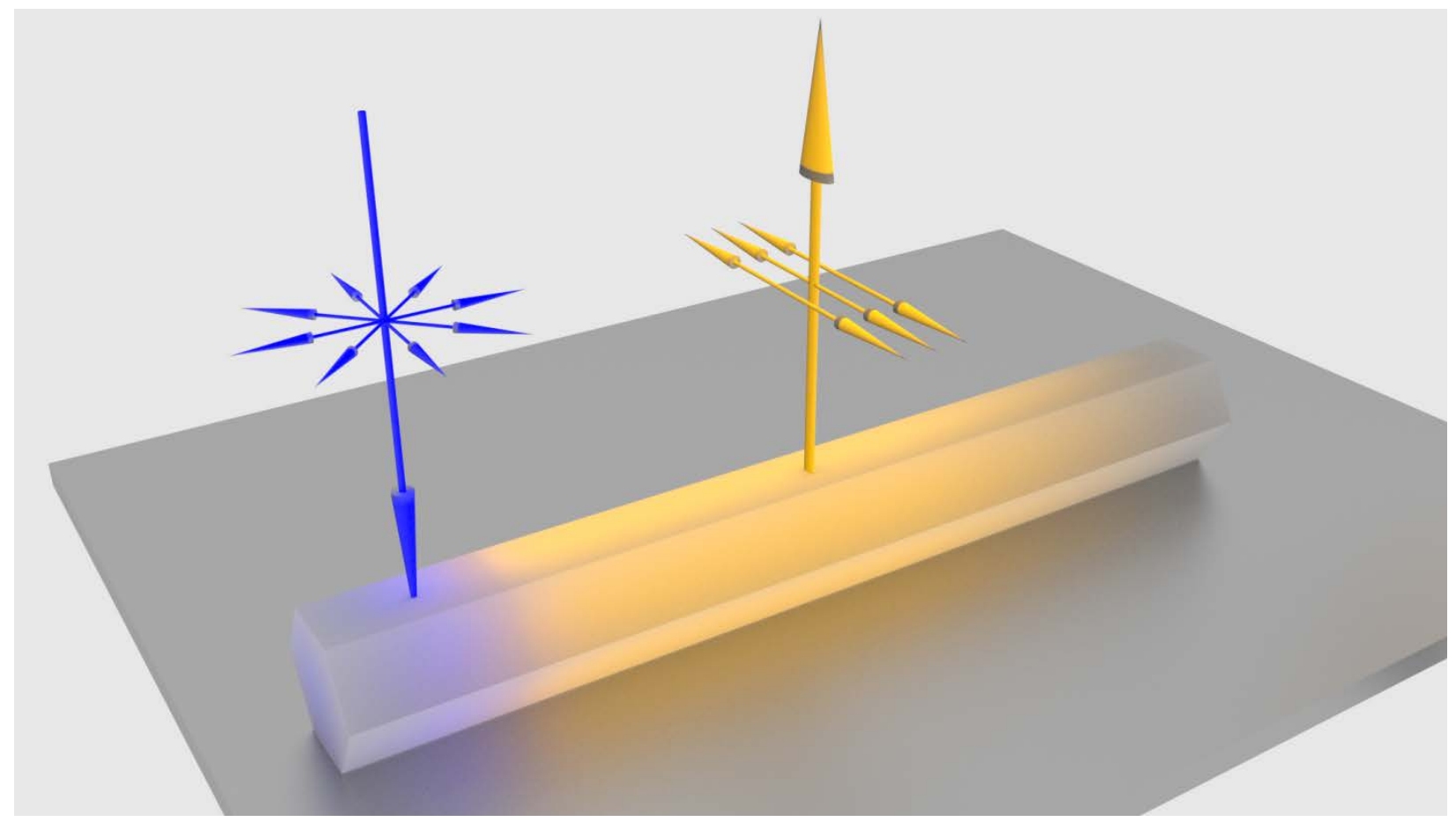

\section{GRAPHICAL TABLE OF CONTENTS.}

We demonstrate that alloying with nitrogen allows one to achieve strong polarization of light emission in individual GaNP and GaP/GaNP core/shell nanowires, which is perpendicular to the 
wire axis even in zinc blende nanowires of various diameters. The observed polarization property is attributed to strong anisotropy of N-related centers responsible for the emission. 